\title{
Kaotik Bir Sistemin Kararlıık Sınır Eğrileri Yöntemi Kullanılarak PI Kontrolör ile Kontrolü
}

\author{
Hatice GÖVERCINN ${ }^{1}$, Vedat ÇELIK ${ }^{2 *}$ \\ ${ }^{1}$ Bilecik Şeyh Edebali Üniversitesi, MYO, Elektrik ve Enerji Bölümü, Elektrik Programı, Bilecik, Türkiye \\ ${ }^{2}$ Fırat Üniversitesi, Mühendislik Fakültesi, Elektrik-Elektronik Mühendisliği Bölümü, Elazığ, Türkiye \\ ${ }^{1}$ hatice.govercin@bilecik.edu.tr, ${ }^{* 2}$ celik@ firat.edu.tr
}

\begin{abstract}
(Geliş/Received: 14/04/2021;
Kabul/Accepted: 03/06/2021)

Öz: Bu makalede, PI kontrolör kullanılarak kaotik Genesio-Tesi sistemin kaos kontrolü amaçlanmıştır ve PI kontrolörün tasarımı kararlılık sınır eğrileri yöntemi ile sağlanmıştır. Bu amaçla, kaotik davranış gösteren Genesio-Tesi sistemin denge noktalarındaki doğrusal matematiksel modelleri elde edilmiştir. Elde edilen matematiksel modellerden orijindeki denge noktasının modeline PI kontrolör eklenmiş ve yeni sistemin ilgili denge noktasını kararlı hale getirecek kontrolör parametre seçimi gerçekleştirilmiştir. Denge noktasını kararlı hale getirecek kontrolör parametrelerinin belirlenmesi için kararlılık sınır eğrileri yöntemi kullanılmıştır. Son olarak, denge noktasını kararlı ve kararsız hale getirecek kontrolör parametreleri için MATLAB/Simulink ortamında sistemin benzetim sonuçları elde edilmiş ve böylece sonuçların doğruluğu gösterilmiştir.
\end{abstract}

Anahtar kelimeler: Kaos kontrol, PI kontrolör, Kaotik Genesio-Tesi sistem, Kararlılık sınır eğrileri yöntemi.

\section{Control of a Chaotic System with PI Controller by Using Stability Boundary Locus Method}

\begin{abstract}
In this paper, chaos control of chaotic Genesio-Tesi system is aimed by using PI controller and the design of PI controller is provided by stability boundary locus method. For this purpose, the linear mathematical models of an equilibrium points of the Genesio-Tesi system showing chaotic behavior is obtained. PI controller is added to the model of the equilibrium point at the origin, which is one of the mathematical models obtained, and the controller parameter choose is performed to stabilize the relevant equilibrium point of the system. The stability boundary locus method is used to determine the controller parameters to stabilize the equilibrium point. Finally, simulation results of the system are obtained in MATLAB / Simulink environment for controller parameters become the equilibrium point stable and unstable, and thus, the accuracy of the results is shown.
\end{abstract}

Key words: Chaos control, PI controller, Chaotic Genesio-Tesi system, Stability boundary locus method.

\section{Giriş}

Kontrol sistemlerinde kullanılan PID kontrolörlerin endüstride yaygın bir şekilde kullanılma nedeni dayanıklı performans ve basit yapıya sahip olmalarıdır. PID kontrolörler genellikle pratik uygulamalarda türevsel kısmının sık kullanılmaması sebebiyle PI şeklindedirler [1]. PID tipi kontrolörlerden biri olan PI kontrolörler, oldukça basit ve birçok kontrol sistemi için çok iyi sonuçlar sağlayan yapı olarak iki parametreye sahip bir kontrolördür. Bir kontrol sisteminde temel amaçlardan biri sistemin kararlılı̆̆ını sağlamak olduğundan dolayı kontrolörler tasarlanırken istenilen sistem performansını sağlamadan önce sistemin kararlı bölgede tutulmasını sağlayacak kontrolör tasarımlarının gerçekleştirilmesi gereklidir. Kontrol sistemlerinde sistemi kararlı hale getirecek tasarım yöntemlerinden biri de kararlılık sınır eğrileri yöntemidir [1-2]. Bu yöntem frekans tabanlı grafiksel bir metottur ve sistem parametrelerinin yanı sıra kontrolör parametrelerinin kararlılık uzayını belirleyebilmeyi de sağlamaktadır. Bu yöntemle tam ve kesir dereceli PID tipi kontrolörlerin doğrusal sistemlerin kararlılığını sağlayacak şekilde tasarımının, başarılı bir şekilde geçekleştirildiği gösterilmiştir [2-5].

Kaos, doğrusal olmayan deterministik bir sistemde gürültü benzeri geniş güç spektrumuna sahip ve başlangıç şartlarına hassas bağlı düzensiz davranışlar olarak tanımlanmaktadır [6]. Bilimsel anlamda ilk defa 20. yüzyılın başlarında Fransız filozof Henry Poincare tarafından astronomi çalışmalarında, karmaşık bir sistemin kararlılı̆̆ ile ilgili olan kısımlarda kullanılmışıı [7]. Bir meteorolog olan Lorenz'in 1963 yılında sıvılardaki türbülans hareketi ile ilgili bir çalışma yapması ile kaos konusunda bilimsel anlamdaki ilk araştırmayı gerçekleştirilmiştir [8]. Kaotik davranış gösteren sistemlerden biri de Genesio-Tesi sistemidir [9]. Bu davranış türünün güvenli haberleşme gibi istendiği uygulama alanları olduğu gibi kontrol sistemleri gibi bu davranışın gözlemlenmesinin istenmediği alanlarda vardır. Bu yüzden [10]'da kaos kontrol fikri ilk defa ortaya atılmış ve kaos kontrol önemli

\footnotetext{
* Sorumlu yazar: celik@ firat.edu.tr. Yazarların ORCID Numarası: ${ }^{1}$ 0000-0001-5008-8870, ${ }^{2}$ 0000-0001-8870-8465
} 
bir çalışma alanı haline gelmiştir [11]. Kaos kontrolde temel amaç kaotik yörüngeleri bir denge noktasına taşımak veya kararlı bir yörüngede tutmaktır. Bu amacı gerçekleştirmeye yönelik bazı çalışmalar söyle sıralanabilir: [12]'de zaman gecikmeli geri besleme kullanılarak, [13]'de kaotik davranış gösteren ekonomik bir modelin durum değişkenleri ve sistem parametreleri kullanılarak, [14]'de birden fazla geri besleme kullanılarak, [15]'de kayma kipli kontrol yöntemi kullanılarak, [16]'da meta sezgisel bir optimizasyon yöntem kullanılarak ve [17]'de kesir dereceli PI kontrolör kullanılarak kaos kontrol işlemi gerçekleştirilmiştir. Yapılan çalışmalara bakıldığında, eğer kaotik sistemin sistem parametrelerine ve durum değişkenlerine pratikte erişim mümkün değilse PI kontrolör kullanımı kaos kontrolü mümkün kılabilir. Bu makalede, literatürde ilk defa doğrusal sistemler için önerilen kararlılık sınır eğrileri yöntemi kullanılarak doğrusal olmayan yapıdaki kaotik davranış gösteren Genesio-Tesi sistemin PI kontrolör kullanarak bu davranıştan kurtarılması amaçlanmıştır. Bu girişle birlikte ikinci bölümde kararlılık sınır eğrileri yöntemiyle doğrusal sistemler için PI kontrolör tasarımı verilmiştir. Üçüncü bölümde kaotik Genesio-Tesi sistem verilmiş ve dördüncü bölümde kararlılık sınır eğrileri yöntemiyle PI kontrolör kullanılarak kaotik Genesio-Tesi sistemin kaos kontrolünün gerçekleştirilmesi MATLAB/Simulink ortamındaki benzetim sonuçlarıyla birlikte sunulmuştur. Sonuçlar bölümde ise elde edilen sonuçlar özetlenip yorumlanmıştır.

\section{Kararlıık Sınır Eğrileri Yöntemi ile PI kontrolör Tasarımı}

Bu bölümde, PI kontrolörlü doğrusal kontrol sistemleri için [2]'de önerilen kararlılık sınır eğrileri yöntemi verilmiş ve kararlılık için PI kontrolör parametre uzayının nasıl elde edileceği sunulmuştur. PI kontrolör tasarımı için Şekil 1'de verilen tek girişli-tek çıkışlı birim geri beslemeli kontrol sistemi ele alınsın.

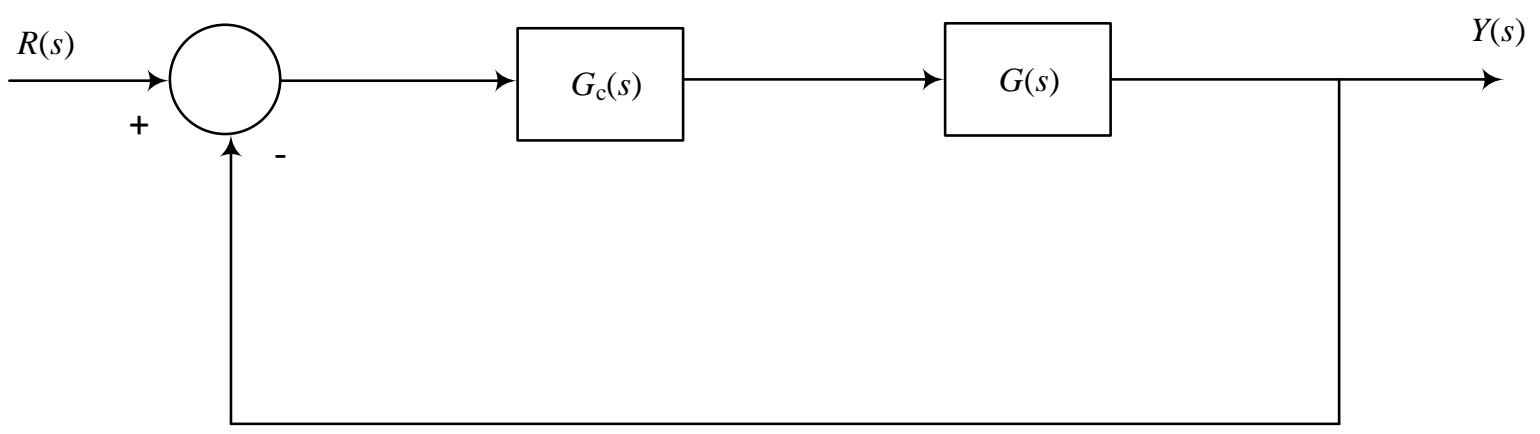

Şekil 1. Tek girişli tek çıkışlı birim geri beslemeli kontrol sistemi

Şekil 1'de $G(s)$, denklem (1)'de verilen $N(s)$ pay ve $D(s)$ payda polinomunundan oluşan kontrol edilecek sistemin transfer fonksiyonudur.

$G(s)=\frac{N(s)}{D(s)}$

$G_{C}(s)$, denklem (2)'de verildiği gibi sistemi kontrol edecek bir PI kontrolördür.

$G_{C}(s)=k_{p}+\frac{k_{i}}{s}$

Denklem (2)'de yer alan $k_{p}$ oransal, $k_{i}$ ise integratör kazancını ifade etmektedir.

Denklem (1)'deki sistemde $s=j \omega$ için pay ve payda polinomları reel ve imajiner bileşenlerine ayrıştırılarak denklem (3) elde edilir.

$G(j \omega)=\frac{N_{e}\left(-\omega^{2}\right)+j \omega N_{o}\left(-\omega^{2}\right)}{D_{e}\left(-\omega^{2}\right)+j \omega D_{o}\left(-\omega^{2}\right)}$ 
Burada, $N_{e}$ ve $D_{e}$ ilgili polinomdaki çift dereceli bileşenleri ve $N_{o}$ ve $D_{o}$ ilgili polinomdaki tek dereceli bileşenleri temsil etmektedir. Aynı işlem denklem (2) için yapıldığında aşağıdaki gibi denklem (4) elde edilecektir.

$G_{C}(j \omega)=\frac{j \omega k_{p}+k_{i}}{j \omega}$

Bu durumda $\Delta(j \omega)$ olarak tanımlanan kapalı çevrimli sistemin karakteristik denklemi aşağıdaki gibi elde edilecektir.

$$
\begin{aligned}
& \Delta(j \omega)=\left[k_{i} N_{e}\left(-\omega^{2}\right)-k_{p} \omega^{2} N_{o}\left(-\omega^{2}\right)-\omega^{2} D_{o}\left(-\omega^{2}\right)\right]+j\left[k_{p} \omega N_{e}\left(-\omega^{2}\right)+k_{i} \omega N_{o}\left(-\omega^{2}\right)+\omega D_{e}\left(-\omega^{2}\right)\right] \\
& =0
\end{aligned}
$$

$\Delta(j \omega)$ reel $\left(R_{\Delta}\right)$ ve imajiner $\left(I_{\Delta}\right)$ kısımları sıfıra eşitlendiğinde denklem (6) ve (7) elde edilecektir.

$$
\begin{aligned}
& k_{p} Q(\omega)+k_{i} R(\omega)=X(\omega) \\
& k_{p} S(\omega)+k_{i} U(\omega)=Y(\omega)
\end{aligned}
$$

Burada;

$$
\begin{aligned}
& Q(\omega)=-\omega^{2} N_{o}\left(-\omega^{2}\right), \quad R(\omega)=N_{e}\left(-\omega^{2}\right), \\
& S(\omega)=\omega N_{e}\left(-\omega^{2}\right), \quad U(\omega)=\omega N_{o}\left(-\omega^{2}\right), \\
& X(\omega)=\omega^{2} D_{o}\left(-\omega^{2}\right), \quad Y(\omega)=-\omega D_{e}\left(-\omega^{2}\right)
\end{aligned}
$$

dir. Bu eşitliklerden frekansa bağlı olarak oransal ve integral kazançları sırasıyla denklem (8) ve denklem (9)'daki gibi elde edilir.

$k_{p}=\frac{X(\omega) U(\omega)-Y(\omega) R(\omega)}{Q(\omega) U(\omega)-R(\omega) S(\omega)}$

$k_{i}=\frac{Y(\omega) Q(\omega)-X(\omega) S(\omega)}{Q(\omega) U(\omega)-R(\omega) S(\omega)}$

Karakteristik denkleme göre üç tane sınır vardır. Bunlar karmaşık kök sınırı, reel kök sınırı ve sonsuz kök sınırı olarak adlandırılır [3]. Karmaşık kök sınırı, $\Delta_{\omega}\left(j \omega, k_{p}, k_{i}\right)=0$, reel kök sınırı, $\Delta_{0}\left(0, k_{p}, k_{i}\right)=0$ ve sonsuz kök sınırı, $\Delta_{\infty}\left(\infty, k_{p}, k_{i}\right)=0$ olarak tanımlanır ve bu tanımlardan faydalanılarak belirlenir.

Denklem (8) ve (9) kullanılarak, kararlılık sınır eğrisi, $\ell\left(k_{p}, k_{i}, \omega\right), k_{p}-k_{i}$ düzleminde çizilebilir. Elde edilen bu değişim karmaşık kök sınırıdır. Ayrıca, $k_{i}=0$ doğrusu kararlılık bölgesinin bir diğger sınırıdır çünkü $\Delta(s)$ 'in bir reel kökü $s=0$ 'da imajiner ekseni keser. Bu değişim reel kök sınırı olarak adlandırılır. Dolayısıyla denklem (5)'te $\omega=0$ için $I_{\Delta}$ zaten sıfır olacaktır ve $R_{\Delta}$ sıfıra eşitlenerek $k_{i}=0$ elde edilir. Bu durumda, $k_{i}=$ 0 reel kök sınırı olacaktır. Son olarak sonsuz kök sınırının $k_{p}, k_{i}$ kontrolör parametrelerine bağlı olmadığ karakteristik denklemden görülmektedir. Böylece sistemi kararlı yapacak PI kontrolör parametre uzayı belirlenebilir. Çünkü karmaşık kök sınırı ile $k_{i}=0$ reel kök sınırı parametre düzlemini yani, $k_{p}-k_{i}$ düzlemini kararlı ve kararsız bölgelere böler. Bu bölgelerde seçilecek kontrolör parametre değerleri kullanılarak hangi bölgenin sistemi kararlı yapacak parametreler içerdiği test edilebilir. 


\section{Kaotik Genesio-Tesi Sistem}

Bu bölümde kaotik Genesio-Tesi sistemin matematiksel modeli, denge noktaları ve kaotik davranış gösterdiği sistem parametrelerinde elde edilen durum uzay diyagramı verilecek ve ikili garip çekici formundaki kaotik davranışın oluşma şartından bahsedilecektir. Genesio-Tesi sistemi Şekil 2'de verilen blok diyagramı ile tanımlanmıştır [6].

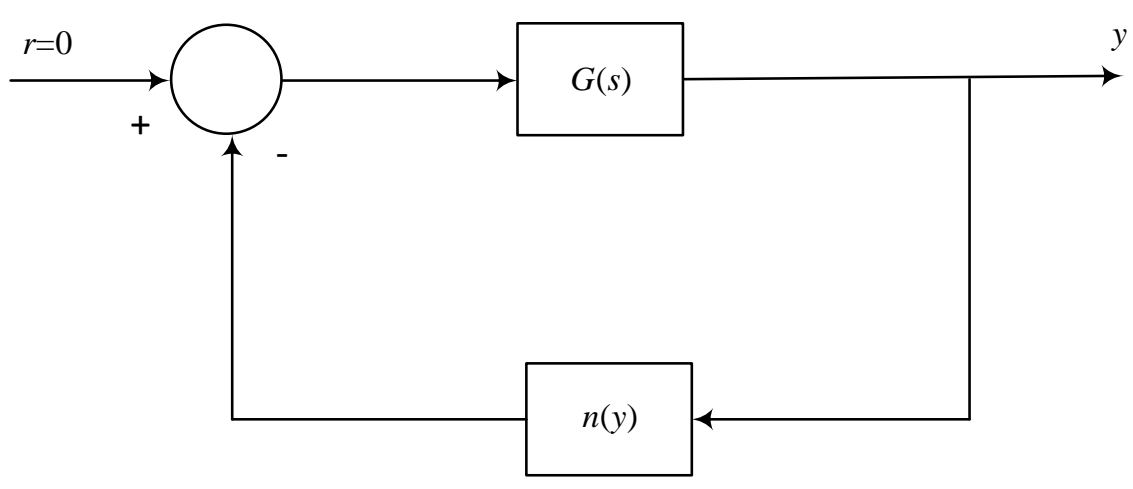

Şekil 2. Kaotik Genesio-Tesi sistemi

Şekil 2'de doğrusal ve doğrusal olmayan sistemden oluşan bir geri beslemeli sistem gösterilmektedir [9]. Bu sistemdeki $G(s)$ ifadesi doğrusal sistem transfer fonksiyonunu, $n(y)$ ifadesi sistemin doğrusal olmayan bileşenini tanımlayan bir fonksiyonu, $r$ giriş fonksiyonunu ve $y$ ise çıkış fonksiyonunu temsil etmektedir.

Genesio-Tesi sistemde $G(s)$ aşağıdaki gibi ifade edilir.

$G(s)=\frac{1}{s^{3}+a s^{2}+b s+c}$

Şekil 2'de yer alan $n(y)$ doğrusal olmayan alt sistem fonksiyonu denklem (11) ile gösterilmektedir.

$n(y)=y^{3}-d y$

Denklem (10) ve denklem (11)'den yararlanarak Şekil 2'de gösterilen geri beslemeli sistemin durum uzay modelini elde etmek için öncelikle denklem (10)'da verilen transfer fonksiyonunun diferansiyel denklem formu aşağıdaki gibi elde edilmiştir.

$\dddot{y}+a \ddot{y}+b \dot{y}+c y=r$

$G(y)+n(y)=0$ olduğundan, Şekil 2'de gösterilmekte olan doğrusal olmayan geri beslemeli sistemin diferansiyel denklem formunda matematiksel ifadesi denklem (13)'teki gibi elde edilecektir.

$\dddot{y}+a \ddot{y}+b \dot{y}+c y+y^{3}-d y=0$

Böylece, denklem (13)’te diferansiyel denklem formundaki doğrusal olmayan sistemin durum uzay modeli $y=x_{1}$ olmak kaydıyla aşağıdaki gibi elde edilir.

$$
\left[\begin{array}{c}
\dot{x_{1}} \\
\dot{x_{2}} \\
\dot{x_{3}}
\end{array}\right]=\left[\begin{array}{ccc}
0 & 1 & 0 \\
0 & 0 & 1 \\
-x_{1}{ }^{2}-c+d & -b & -a
\end{array}\right]\left[\begin{array}{l}
x_{1} \\
x_{2} \\
x_{3}
\end{array}\right]
$$

Dikkat edilirse denklem (13), blok diyagramında gösterildiği gibi özerk bir sistemdir. Bu sistemin denge noktaları durum denklemlerinden $E^{0}(0,0,0), E^{+}(+\sqrt{d-c}, 0,0), E^{-}(-\sqrt{d-c}, 0,0)$ olarak elde edilir. 
Bu sistem, $a=0.2, b=1.5, c=1.2, d=2$ değerleri için kaotik davranış göstermektedir. Şekil 3 'te bu değerler için sistemin MATLAB/Simulink ortamında elde edilmiş durum uzay diyagramı gösterilmektedir.

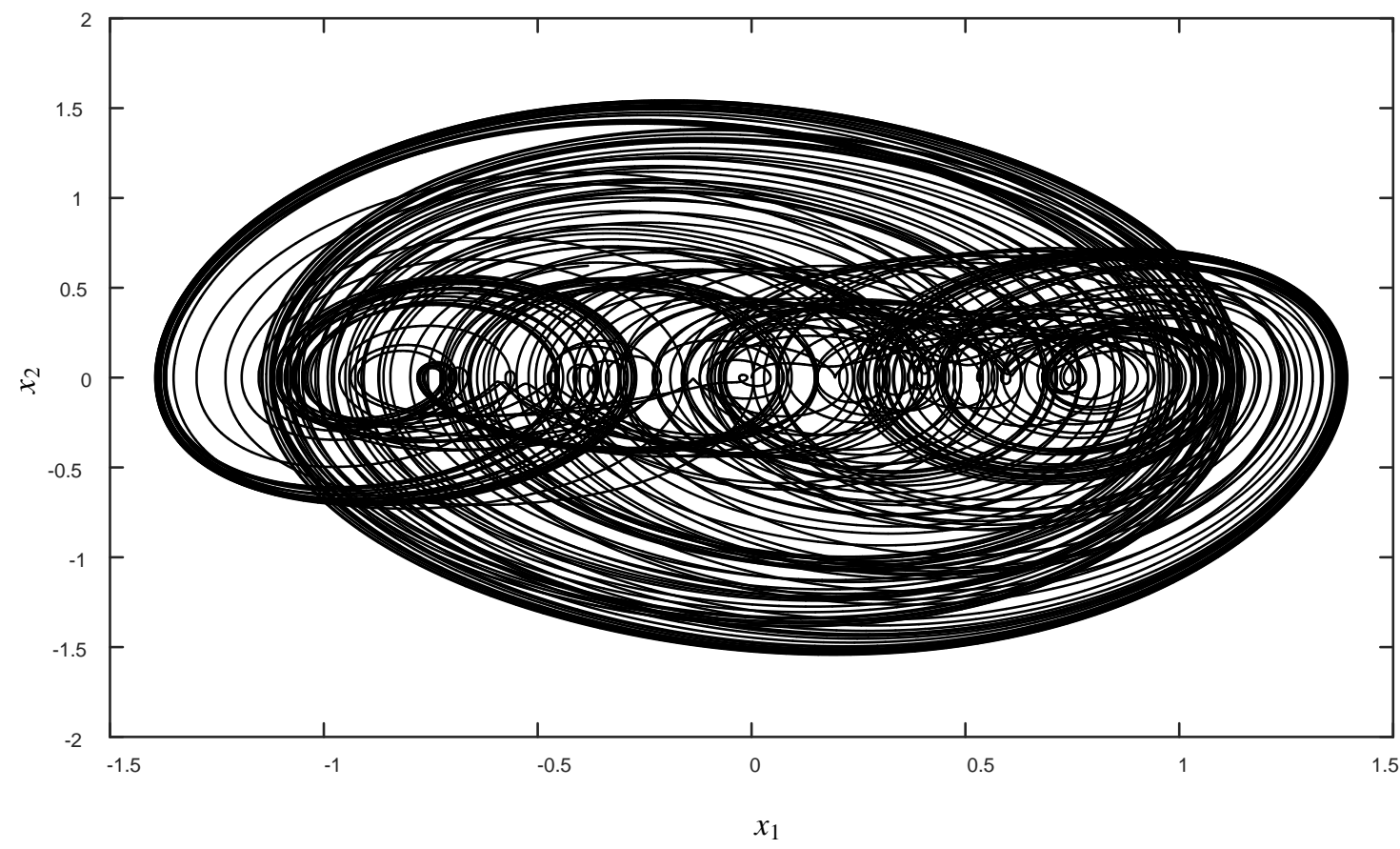

Şekil 3. Kaotik Genesio-Tesi sistemin durum uzay diyagramı

Şekil 3'ten, verilen parametreleri için, sistemin ikili garip çekici formunda kaotik davranış gösterdiği gözlenmektedir. Bu yapıdaki kaotik sistemlerin kaotik davranış gösterebilmesi için orijindeki denge noktasının İndeks 1'in eğer noktası karakteristiğine, diğer denge noktalarının ise İndeks 2'nin eğer noktası karakteristiğine sahip olması gerekir [18].].Indeks 1'in eğer noktası sayesinde oluşan ikili kaotik çekicilerin bağlantısı sağlanmış olur.

\section{Kararlılık Sınır Eğrileri Yöntemi Kullanılarak PI Kontrolör ile Kaotik Genesio-Tesi Sistemin Kontrolü}

Bu bölümde kaotik Genesio-Tesi sistemin kontrolü için gerekli olan kontrolör tasarımı kararlılık sınır eğrileri yöntemiyle gerçekleştirilecek ve tasarlanan kontrolörün kaotik sistem üzerine etkisi simülasyon sonuçlarıyla doğrulanacaktır. Şekil 2'de verilen ve bir önceki bölümde incelenen kaotik Genesio-Tesi sisteme PI kontrolör eklenerek sistemi kaotik davranıştan kurtarılacaktır. Şekil 4'te bu sistemin blok diyagramı gösterilmektedir.

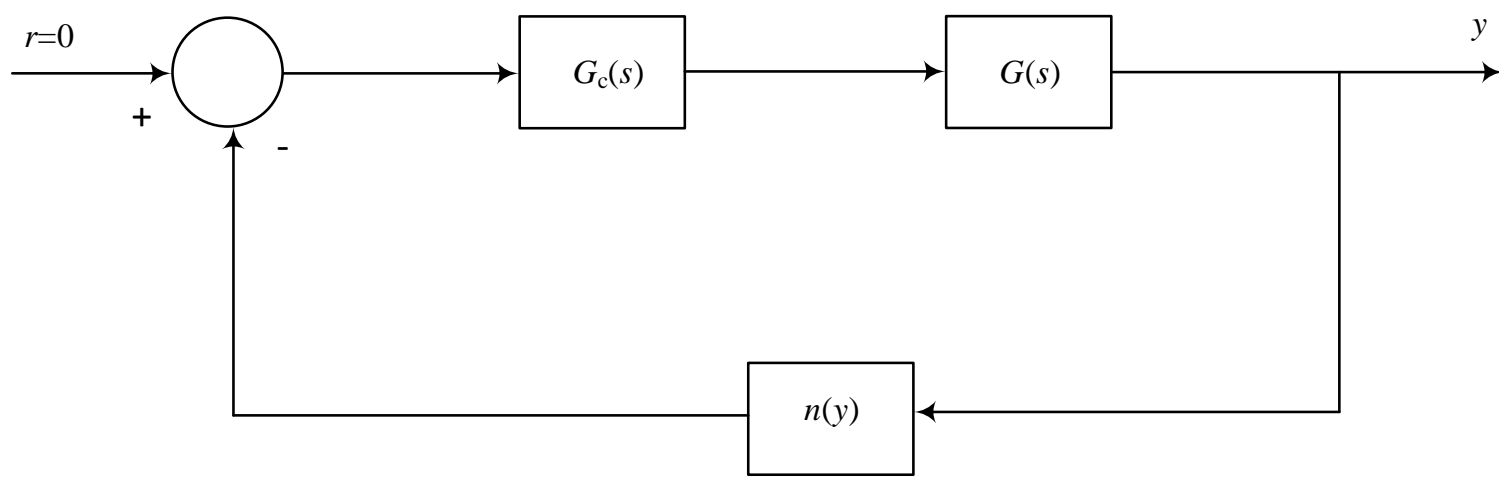

Şekil 4. PI kontrolörlü Genesi-Tesi sistem 
Şekil 4'te gösterilmekte olan geri beslemeli sistemde, kontrol edilecek sistemin transfer fonksiyonu denklem (15)'de, PI kontrolörün transfer foksiyonu denklem (16)'da ve doğrusal olmayan bir fonksiyon olan geri besleme birimi ise denklem (17)'de verilen eşitliklerdir.

$G_{c}(s)=k_{p}+\frac{k_{i}}{s}$

$G(s)=\frac{1}{s^{3}+a s^{2}+b s+c}$

$n(y)=y^{3}-d y=0$

$s$-domeninde analizlerin gerçekleştirilebilmesi için denklem (17)'de verilen doğrusal olmayan fonksiyonun denge noktaları civarında doğrusal modeli elde edilmelidir. Bu amaçla ilk önce denklem (17)'de verilen doğrusal olmayan fonksiyonun denge noktaları bulunmuştur. $\mathrm{Bu}$ denge noktalarının, $y_{1}=0, y_{2,3}= \pm \sqrt{d}$ olduğu görülmektedir. Denklem (17)'nin denge noktaları civarındaki doğrusal modelleri, $y_{1}=0$ denge noktası için $A_{1}=-d$ ve $y_{2,3}= \pm \sqrt{d}$ denge noktaları için $A_{2,3}=2 d$ olarak elde edilir.

Eğer $y_{1}=0$ 'daki denge noktası kaotik davranış için gerekli olan İndeks 1 'in eğer noktası karakteristiğinden kurtarılabilirse sistem kaotik davranıştan kurtarılacaktır. Bu yüzden orijindeki denge noktası kararlı hale getirilmeye çalışılacaktır. Bu durumda, $y_{1}=0$ denge noktasındaki doğrusal modeli kullanılarak Şekil 4 'te verilen sistemin karakteristik denklemi aşağıdaki gibi olacaktır.

$\Delta(s)=1+G_{c}(s) G(s) A_{1}$

Transfer fonksiyonları yerine yazıldığında aşağıdaki karakteristik denklem elde edilecektir.

$\Delta(s)=s^{4}+a s^{3}+b s^{2}+\left(c-d k_{p}\right) s-d k_{i}=0$

Denklem (19)'da $s$ yerine jw yazılarak karakteristik denklemin çözümü gerçekleştirildiğinde Şekil 4'te verilen Genesio-Tesi sistemin $y_{1}=0$ denge noktasındaki kararlılık sınır eğrilerinin elde edilmesini sağlayacak PI kontrolörün integratör ve oransal kazançları sırasıyla aşağıdaki gibi elde edilir.

$k_{i}=\frac{\omega^{4}-b \omega^{2}}{d}$

$k_{p}=\frac{-a \omega^{2}+c}{d}$

Bir önceki bölümde Genesio-Tesi sistemin kaotik davranış gösterdiği, $a=0.2, b=1.5, c=1.2, d=2$, parametreler kullanılarak $y_{1}=0$ 'daki denge noktasını kararlı hale getirecek PI kontrolör kazançlarını elde etmek için kararlılık sınır eğrileri, $\ell\left(k_{p}, k_{i}, \omega\right)$, belirli frekans aralıkları için $\left(k_{p}, k_{i}\right)$ düzleminde denklem (20) ve (21)'den faydalanılarak MATLAB ortamında Şekil 5'deki gibi elde edilmiştir. 


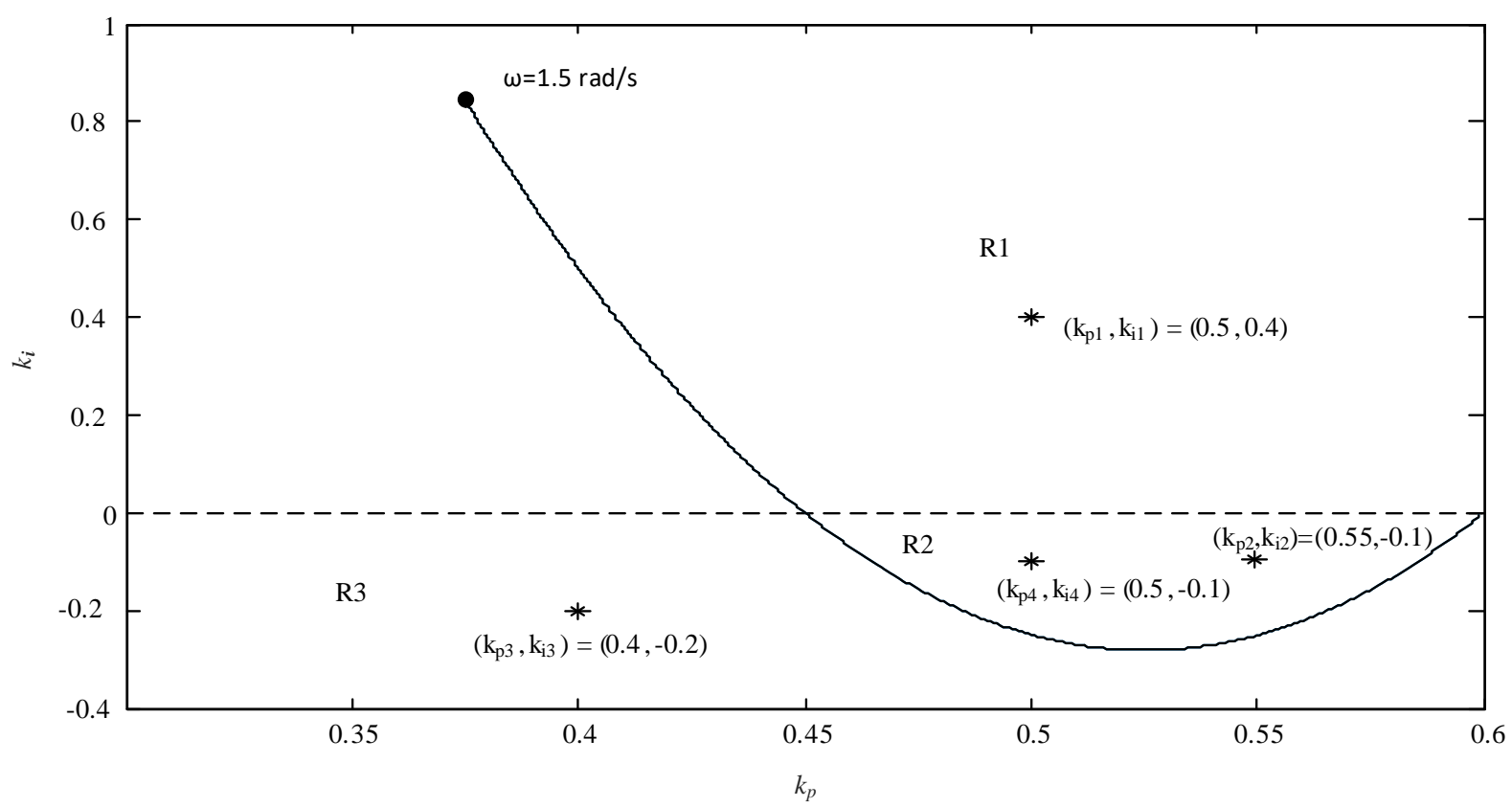

Şekil 5. Şekil 4’te verilen sistemin denge noktasındaki doğrusal modeli için kararlılık sınır eğrisi

Şekil 5'te elde edilen değişim karmaşık kök sınırıdır. $k_{i}=0$ ise reel kök sınırı olacaktır ve kesikli çizgiler ile şekil üzerinde gösterilmiştir. Bu sistem için sonsuz kök $, k_{p}, k_{i}$ parametrelerine bağlı olmadığından sonsuz kök sınırı mevcut değildir. Böylece, karmaşık kök ve reel kök sınırları arasında kalan bölgenin kararlı olması beklenir. Ancak, bu bölgelerin test edilmesi gerekecektir. Bunun için $\left(k_{p}, k_{i}\right)$ düzlemi üzerinde R1, R2 ve R3 olarak adlandırılan üç bölgede test noktaları belirlenmiştir.

Şekil 5'te R1 bölgesi içinde $k_{p 1}=0.5, \quad k_{i 1}=0.4$ test noktası için karakteristik denklem aşağıdaki gibi olacaktır.

$\Delta(s)=s^{4}+0.2 s^{3}+1.5 s^{2}+0.2 s-0.8=0$

Bu durumda, karakteristik denklemin kökleri yani kapalı çevrim sistemin kutupları aşağıdaki gibi elde edilecektir.

$s_{1}=-0.0391+j 1.3813$

$s_{2}=-0.0391-j 1.3813$

$s_{3}=-0.7111$

$s_{4}=0.5892$

Dikkat edilirse, $s_{4}$ sağ yarı $s$-düzleminde bir kutba karşılık gelmektedir. Bu yüzden, R1 bölgesi içindeki $k_{p 1}=0.5, k_{i 1}=0.4$ test noktası kararsızdır. Bu sonuç R1 bölgesinin kararsız parametre uzayına karşılık geldiği anlamına gelmektedir. Şekil 6'da R1 bölgesi içinde seçilen test noktası için MATLAB/Simulink ortamında sistem çıkış işaretinin, kontrolörün belirli bir zamanda aktif edilmesi durumuna göre değişimi elde edilmiştir. 




Şekil 6. R1 bölgesi içinde seçilen $k_{p 1}=0.5, k_{i 1}=0.4$ test noktası için sistemin zaman cevab1

Burada 150.s'de PI kontrolör devreye girmesine rağmen sistemin kaotik davranıştan kararsız bir davranışa geçtiği görülmektedir.

Şekil 5'teki R2 bölgesi içinde $k_{p 2}=0.55, k_{i 2}=-0.1$ test noktası için karakteristik denklem aşağıdaki gibi olacaktır.

$\Delta(s)=s^{4}+0.2 s^{3}+1.5 s^{2}+0.1 s+0.2=0$

Bu durumda, sistemin kutupları aşağıdaki gibi elde edilecektir.

$s_{1}=-0.0706+j 1.1566$

$s_{2}=-0.0706-j 1.1566$

$s_{3}=-0.0294+j 0.3848$

$s_{4}=-0.0294-j 0.3848$

Dikkat edilirse, tüm kutuplar sol yarı $s$-düzlemindedir. Bu yüzden, R2 bölgesi içindeki $k_{p 2}=0.55, k_{i 2}=$ -0.1 test noktası kararlıdır. Bu sonuç R2 bölgesinin kararlı parametre uzayına karşılık geldiği anlamına gelmektedir. Şekil 7'de R2 bölgesi içinde seçilen kontrolör parametreleri için MATLAB/Simulink ortamında sistem çıkış işaretinin, kontrolörün belirli bir zamanda aktif edilmesi durumuna göre değişimi elde edilmiştir. 


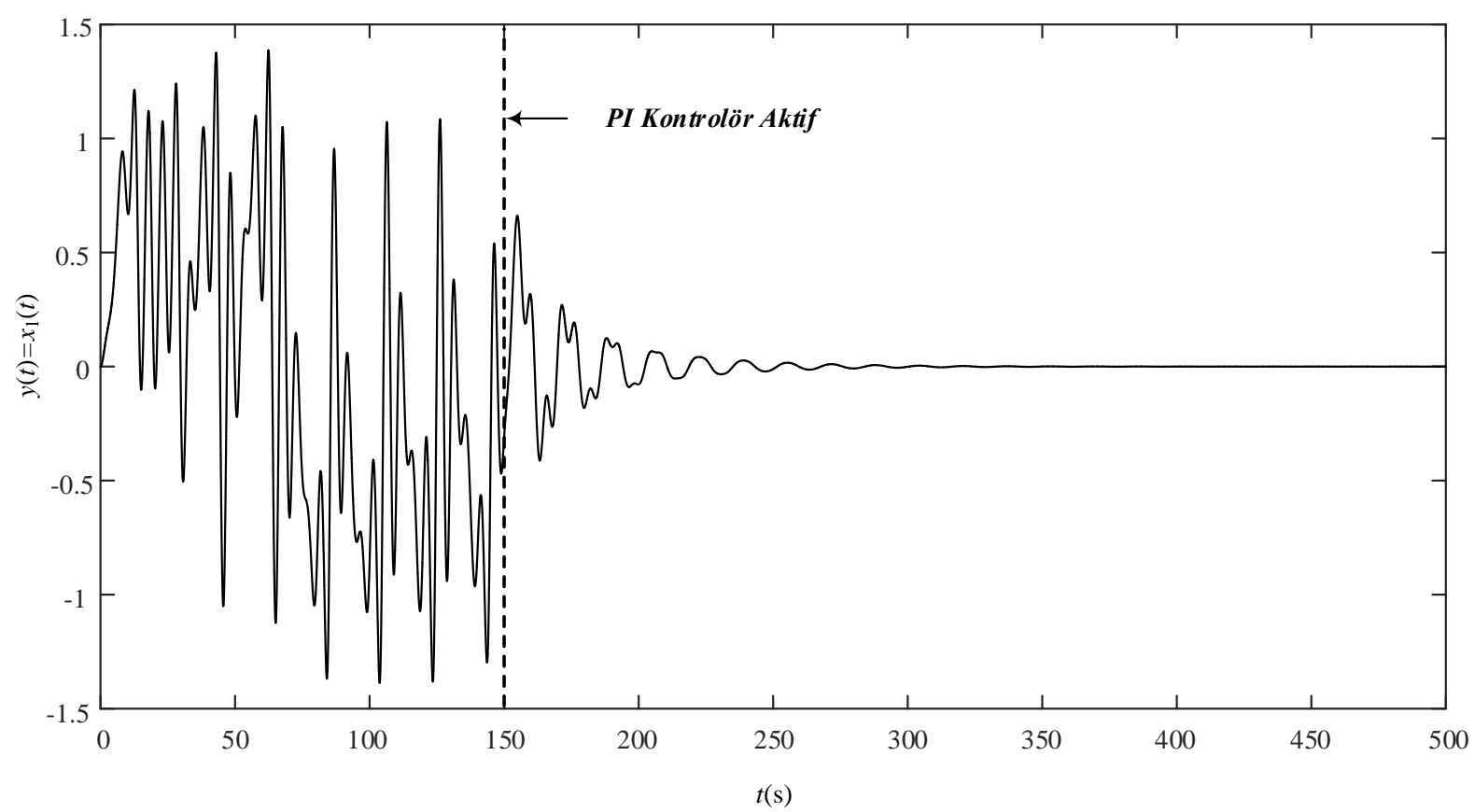

Şekil 7. R2 Bölgesi içinde seçilen $k_{p 2}=0.55, k_{i 2}=-0.1$ test noktas1 için sistemin zaman cevab1

Şekil 7 incelendiğinde 150. saniyede PI kontrolörün devreye girdiği ve sistemdeki kaotik davranışının kontrolünün bu andan itibaren sağlanarak sistem çıkış işaretinin geçici durum davranışından sonra zamana göre kararlı bir davranış gösterdiği görülmektedir.

Şekil 5'teki R3 bölgesi içinde $k_{p 3}=0.4, k_{i 3}=-0.2$ test noktası için karakteristik denklem aşağıdaki gibi olacaktır.

$\Delta(s)=s^{4}+0.2 s^{3}+1.5 s^{2}+0.4 s+0.4=0$

Bu durumda, sistemin kutupları aşağıdaki gibi elde edilecektir.

$s_{1}=0.0833+j 1.1095$

$s_{2}=0.0833-j 1.1095$

$s_{3}=-0.1833+j 0.5381$

$s_{4}=-0.1833-j 0.5381$

Elde edilen kutuplara bakıldığında, iki kutup sağ yarı $s$-düzlemindedir. Bu yüzden, R3 bölgesi içindeki $k_{p 3}=$ $0.4, k_{i 3}=-0.2$ test noktası kararsızdır. Bu sonuç R3 bölgesinin kararsız parametre uzayına karşılık geldiği anlamına gelmektedir. Şekil 8'de R3 bölgesi içinde seçilen kontrolör parametreleri için MATLAB/Simulink ortamında sistem çıkış işaretinin, kontrolörün belirli bir zamanda aktif edilmesi durumuna göre değişimi elde edilmiştir. 


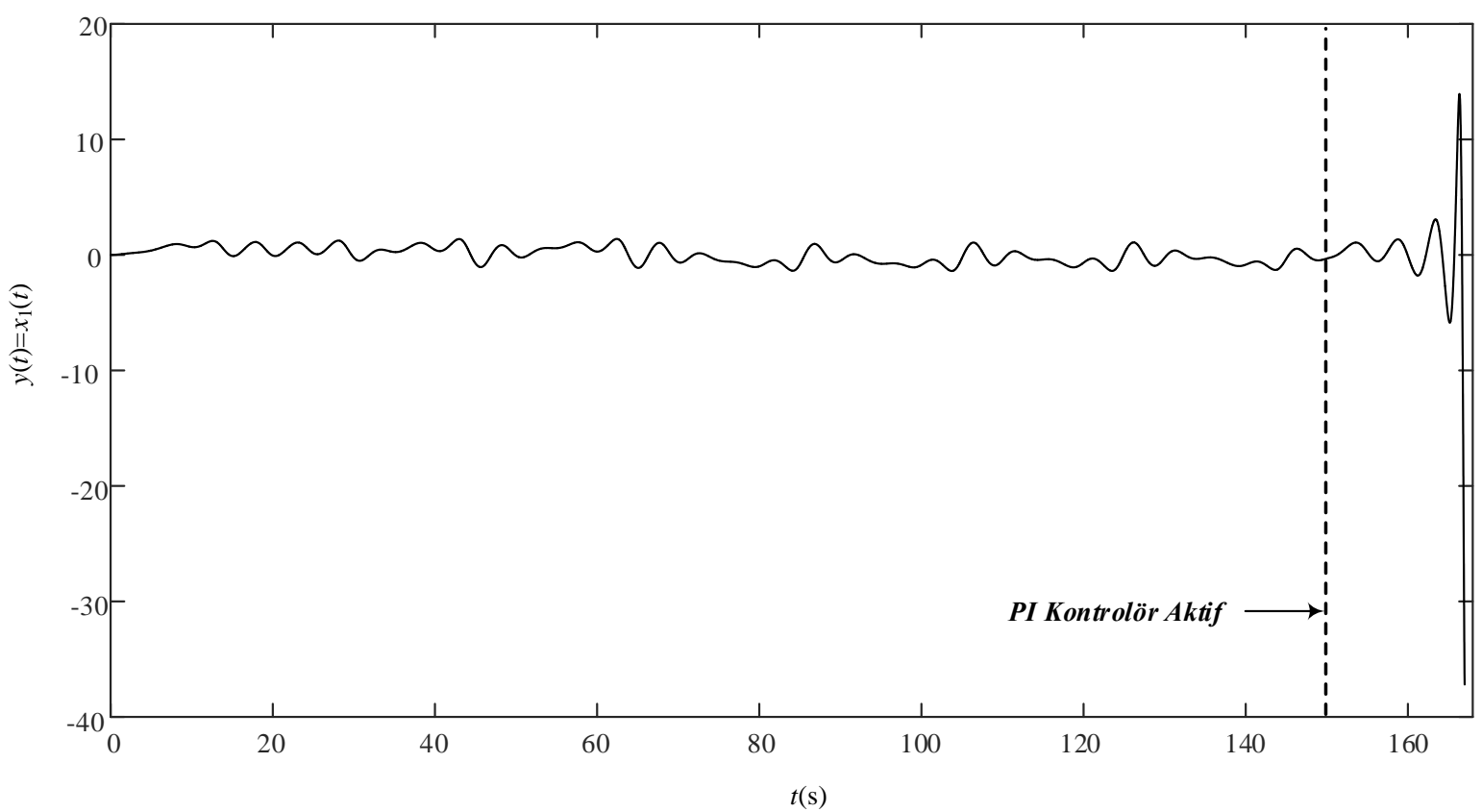

Şekil 8. R3 Bölgesi içinde seçilen $k_{p 3}=0.4, k_{i 3}=-0.2$ test noktası için sistemin zaman cevab1

Şekil 8'de görüldüğü gibi 150. s'de PI kontrolör devreye girmesine rağmen sistemin kaotik davranıştan kararsız bir davranışa geçtĭgi görülmektedir.

Son olarak, Şekil 5 'teki R2 bölgesi içerisinde farklı bir nokta olan $k_{p 4}=0.5, k_{i 4}=-0.1$ için karakteristik denklem aşağıdaki gibi olacaktır.

$\Delta(s)=s^{4}+0.2 s^{3}+1.5 s^{2}+0.2 s+0.2=0$

Bu kontrolör parametreleri için sistemin kutupları aşağıdaki gibi elde edilecektir.

$s_{1}=-0.0287+j 1.1585$,

$s_{2}=-0.0287-j 1.1585$

$s_{3}=-0.0713+j 0.3793$

$s_{4}=-0.0713-j 0.3793$

Tüm kutuplar sol yar1 $s$-düzleminde olduğu için R2 bölgesi içindeki $k_{p 4}=0.5, k_{i 4}=-0.1$ noktası kararlıdır. Bu sonuç R2 bölgesinin kararlı parametre uzayına karşılık geldiğini bir kez daha doğrulamaktadır. Şekil 9'da R2 bölgesi içinde seçilen kontrolör parametreleri için MATLAB/Simulink ortamında sistem çıkış işaretinin, kontrolörün belirli bir zamanda aktif edilmesi durumuna göre değişimi elde edilmiştir. 


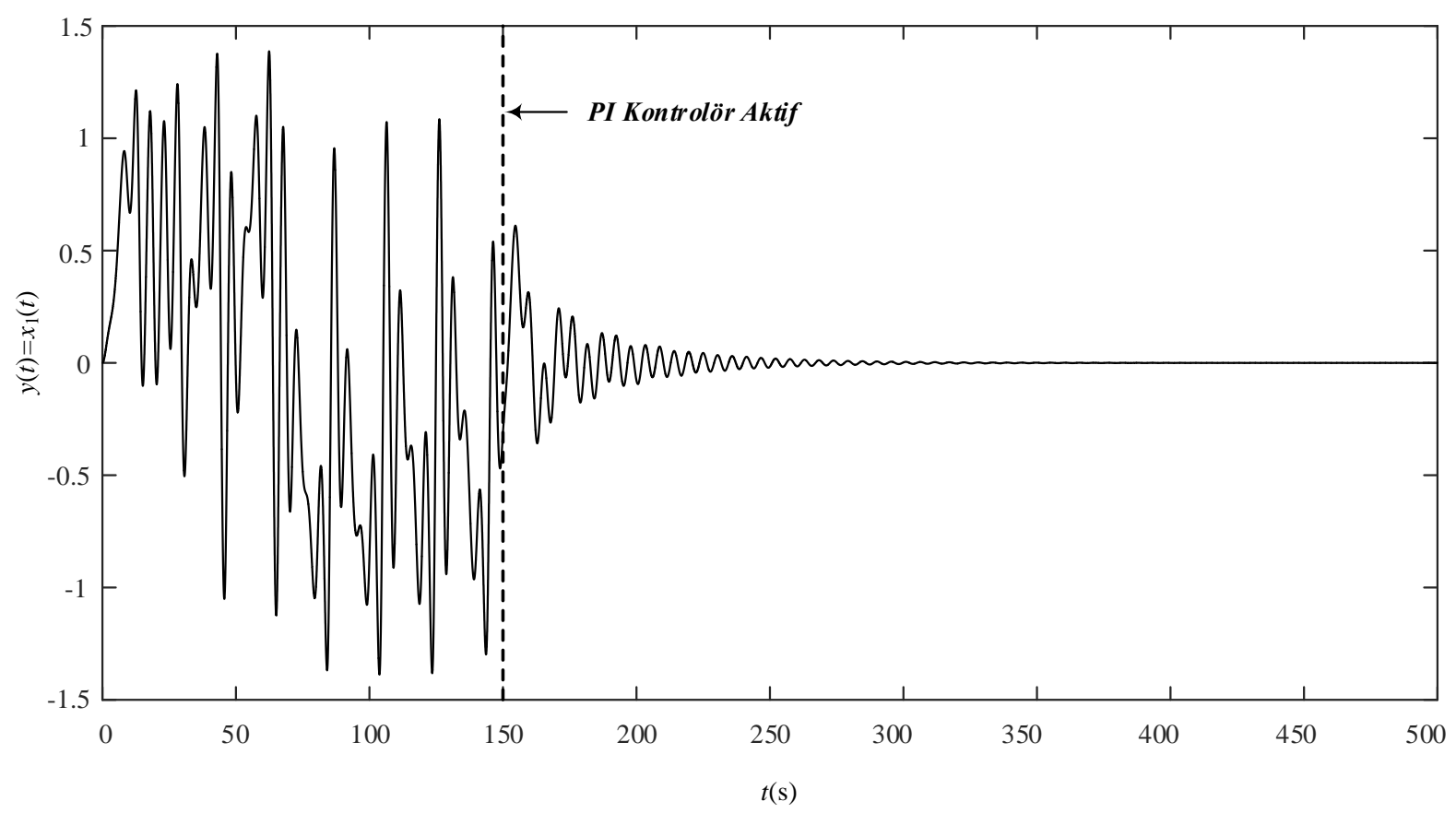

Şekil 9. R2 Bölgesi içinde seçilen $k_{p 4}=0.5, k_{i 4}=-0.1$ test noktası için sistemin zaman cevab1

Şekil 9'da 150. saniye de PI kontrolörün devreye girdiği ve sistemin kaotik davranışının kontrolünün bu andan itibaren sağlanarak sistem çıkış işaretinin zamana göre kararlı bir davranış gösterdiği görülmektedir. Dikkat edilirse, sistemin geçici durum davranışı sönümlü bir osilasyon yaparak sıfıra girmektedir. Bunun sebebi sistemin dominant kutuplarının kompleks eşlenik olmasından kaynaklanmaktadır. Bu durum Şekil 7'de elde edilen değişim için de geçerlidir.

Elde edilen sonuçların genel bir değerlendirmesi yapıldığında $k_{p}-k_{i}$ düzleminde elde edilen karmaşık kök sınırı, $\ell\left(k_{p}, k_{i}, \omega\right)$ ve reel kök sınırı, $k_{i}=0$ arasında kalan R2 bölgesi içerisinde seçilecek herhangi bir PI kontrolör parametre değeri için kaotik davranış gösteren Genesio-Tesi sistemin kaotik davranıştan kurtarılabileceği görülmüştür.

\section{Sonuç}

Bu makalede, kaotik davranış gösteren Genesi-Tesi sistem PI kontrolör kullanılarak kaotik davranıştan kurtarmak amaçlanmıştır. Bu amaçla, kaotik davranış gösteren Genesi-Tesi sistemin orijindeki denge noktası için doğrusal modeli elde edilmiş ve elde edilen model kullanılarak orijindeki denge noktasını kararlı hale getirecek kontrolör parametreleri belirlenmiştir. Denge noktasını kararlı hale getirecek kontrolör parametrelerinin belirlenmesi için kararlılık sınır eğrileri yöntemi kullanılmıştır. Elde edilen sonuçların doğruluğunu göstermek için MATLAB/Simulink ortamında sistemin benzetim sonuçları ayrıca sunulmuştur. Bu sonuçlara göre kaotik sistemi kontrol etmek için belirlenen denge noktasını kararlı hale getirecek PI kontrolör parametreleri için sistem kaotik davranıştan kurtulurken diğer kontrolör parametreleri için sistem kararsız bir davranış göstermiştir. Ayrıca elde edilen sonuçlar, doğrusal sistemlerin kontrolü için önerilen kararlılık sınır eğrileri yönteminin doğrusal olmayan sistemlere de uygulanabileceğini ve mevcut kaos kontrol yöntemlerine bir alternatif olabileceğini de göstermiştir.

\section{Kaynaklar}

[1] Astrom K.J. and Hagglund T., 2001. The Future of PID control. Control Engineerig Practice, 9, 1163-1175.

[2] Tan, N., Kaya, I., Yeroglu, C., and Atherton, D. P., 2006. Computation of stabilizing PI and PID controllers using the stability boundary locus, Energy Conversion and Management, 47, 3045-3058.

[3] Hamamci, S. E., 2007. An algorithm for stabilization of fractional-order time delay systems using fractional-order PID controllers,IEEE Trans Automat Contr, 52(10), 1964-1969. 
[4] Özdemir, M. T., Çelik, V., 2017. Stability analysis of the automatic voltage regulation system with PI controller, Sak. Univ. J. Sci., 21(4), 698-705.

[5] Çelik, V., Özdemir, M.T., Bayrak, G., 2017. The effects on stability region of the fractional-order PI controller for onearea time-delayed loadfrequency control systems, Trans Inst Meas Contr, 39(10), 1509-1521.

[6] Strogatz, S. H., 1994. Nonlinear Dynamics and Chaos, Westview Press.

[7] Isham, V., 1992. Discussion on the meeting on chaos, Journal of Royal Statistical Society, 54(2), 451-454.

[8] Lorenz, E.N., 1963. Deterministic non-periodic flow, Journal of the Atmospheric Sciences, 20(2), 130-141.

[9] Genesio, R ., Tesi , A., 1991.Chaos prediction in nonlinear feedback systems, IEE proceedings D-control theory and applications, 138 (4), 313-320.

[10] Ott, E., Grebogi, C., Yorke, J. A., 1990. Controlling chaos, Phys. Rev. Lett., 64, 1196 -1199.

[11] Kapitaniak T., 1996. Controlling Chaos: Theoretical and practical methods in nonlinear dynamics, Academic Press Inc.

[12] Pyragas K., 1992. Continuous control of chaos by self-controlling feedback. Phys Lett A, 170, 421-428.

[13] Holyst, J. A., Urbanowicz K., 2000. Chaos control in economical model by time-delayed feedback method, Physica A, 287, 587-598.

[14] Yassen, M.T., 2003. Chaos control of Chen chaotic dynamical system, Chaos, Solitons and Fractals, 15, $271-283$.

[15] Nazzal, J. M., Natsheh, A. N., 2007. Chaos control using sliding-mode theory, Chaos, Solitons and Fractals, 33, 695-702.

[16] Zelinka, I., Senkerik, R., Navratil, E., (2009). Investigation on evolutionary optimization of chaos control, Chaos, Solitons and Fractals, 40, 111-129.

[17] Çelik, V., Demir Y., 2010. Effects on the chaotic system of fractional order PI ${ }^{\alpha}$ controller, Nonlinear Dynam, 59(1-2), 143-159.

[18] Tavazoei, M. S., Haeri, M., 2008. A necessary condition for double scroll attractor existence in fractional-order systems, Physics Letters A, 367(1-2),102-113. 